\title{
Obtención del hormigón apropiado a terrenos $y / o$ aguas agresivas diversas mediante la norma TGL 11357
}

RAFAEL TALERO

Ledo. en Ciencias Químicas IETCC/CSIC

\begin{abstract}
RES UMEN
La norma TGL 11357 especifica la utilización expresa de cementos:

- Portland con un contenido de $C_{3} A$ del $0 \%$ al $5 \%$, o

- Siderúrgicos Sobresulfatados (con condiciones), o

- Aluminosos (con condiciones)

cuando la agresividad del agua y/o terreno agresivo que vaya a estar en contacto con la obra de hormigón sea del tipo sulfático, entre otras.

Existen muchos paises que no disponen de tal tipo tan especifico de cementos para poder cumplimentarla, y mediante el presente articulo, al ampliar fundadamente la gama de variabilidad de aquellos adecuados citados, a sólo determinados puzolánicos, se les facilitará bastante más la posible cumplimentación del espiritu de la norma en cuestión, para alcanzar idéntico objetivo, o sea, un hormigón de cemento con adiciones puzolánicas adecuadas ( $y / o$ escorias siderometalúrgicas adecuadas), resistente al mencionado ataque agresivo.
\end{abstract}

\author{
$S U M M A R Y$
}

TGL 11357 Norm specifies the express use of the cement:

- Portland with a content between $0 \%$ and $5 \%$ of $C_{3} A$, or

- Supersulphathed iron foundry (with conditions), or

- Aluminates (with conditions)

when the agressiveness of the water and/or of an agressive sulphatic ground (among others) will be touching the concrete work.

There are countries where there are not such a specific kind of cement, being unable to execute this norm. But by means of this study, which enlarge with good reason the range of those appropiate composites to defined puzzolans, it would be easier to keep the meaning o this norm, to reach identical purpose, that is, a cement concrete with appropriate puzzolanic additions (and/or appropriate iron slags) resistant to the mentioned agressiveness.

Como es sabido la norma de Alemania Oriental (DDR) TGL 11357 (1), está destinada fundamentalmente, a la calificación del grado de agresividad de un agua y/o terreno agresivo que vaya o pueda estar, en contacto con un hormigón cualquiera, sea cual fuere la forma estructural que este adoptare en la realidad, así como también la elección correspondiente del mismo, y sobre todo de los componentes fundamentales, conglomerante hidráulico y árido, entre otros, en cada caso concreto.

De la lectura y estudio detallado de la misma se obtienen, como conclusiones fundamentales, las siguientes:

1. ${ }^{\text {a }) ~ Q u e ~ t o d o s ~ y ~ c a d a ~ u n o ~ d e ~ l o s ~ d i s t i n t o s ~ g r a d o s ~ d e ~ a g r e s i v i d a d, ~ d e l ~ I ~ a l ~ V, ~ c o n ~ q u e ~ p u e d e ~}$ calificar esta norma al agua y/o terreno agresivo problema, se pueden obtener de forma individualizada,

- o por la columna A, véase Tabla 2,

- o por la columna B, véase Tabla 2 , 
- o por la columna C, véase Tabla 2,

lo cual implicará la presencia ineludible de iones sulfato $\mathrm{SO}_{4}^{2-}$ en el caso de la columna $\mathrm{C}$ y la presencia posible en el caso de la B.

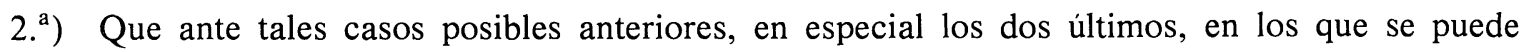
contar con la presencia de iones sulfato, $\mathrm{SO}_{4}^{2-}$, en el agua y/o terreno problema, los cementos especificados, en su caso, por dicha norma son los "cementos sulforresistentes apropiados", es decir,

- los cementos Portland con menos de un $5 \%$ de aluminato tricálcico $\left(\mathrm{C}_{3} \mathrm{~A}\right)$,

- los cementos Portland exentos de aluminato tricálcico,

- los cementos Siderúrgicos Sobresulfatados (con limitaciones específicas) (2) y

- los cementos Aluminosos (con limitaciones especificas),

aconsejando además la norma citada "la eficacia de la adición de PUZOLANAS, independientemente del tipo de cemento empleado y de las adiciones de otros materiales porque la práctica así lo ha demostrado...", según la norma en cuestión.

Pues bien, en vista de:

- que muchos países no pueden disponer de tales cementos tan específicos aconsejados por la norma, así como tampoco de escorias siderúrgicas, y si en cambio y solamente de cementos portland de no elevada resistencia al ataque de los iones sulfato y de puzolanas, y de

- los resultados, conclusiones y aplicaciones obtenidos en este Instituto por el Lcdo. Rafael Talero Morales*, los cuales demuestran la posibilidad real de utilización de determinados cementos portland con adiciones. PA. (3) y/o puzolánicos PUZ (3), contra el mencionado ataque sulfático.

Existe la posibilidad real de poder alcanzar también los objetivos perseguidos por la citada norma, merced a tales aplicaciones, con lo cual se facilitará su cumplimentación, en el fondo que no en la forma, por los países anteriores citados.

Por lo tanto y según lo anterior, en el presente artículo, simplemente se dará una panorámica general resumida de la norma en cuestión - pues para más detalle y abundamiento sobre la misma remítase el lector a la Bibliografía correspondiente- haciendo, eso sí, hincapié en el apartado correspondiente a qué tipo y clase de cemento será el indicado en cada caso, según lo anterior y en especial la luz arrojada por el trabajo del Lcdo. R. Talero, antes citado.

Por todo lo cual el presente resumen constará de cuatro partes fundamentales:

A. NORMA TGL 11357, "HORMIGONES EN AGUAS AGRESIVAS”, dada como de gran interés para obtener un buen comportamiento del hormigón frente a las aguas agresivas, a fin de preparar aquel que fuese más idóneo para resistir el ataque de las mismas.

Ideas fundamentales para realizar una adecuada utilización de la misma:

1. Campos de utilización de la presente norma.

\footnotetext{
* Trabajos de Tesis Doctoral, los cuales se encuentran actualmente en fase de redacción y composición para su lectura y posterior publicación.
} 
2. Examen de dichos terrenos y/o aguas más o menos agresivas.

2.1. Toma de muestras.

2.2. Análisis o determinaciones específicas de cada tipo de terreno y/o agua agresiva posible.

3. Determinación de los siguientes conceptos referentes al agua o terreno problema:

3.1. Grado de agresividad potencial inicial o teórica $\left(G A P_{i}\right)$.

3.2. Grado de agresividad potencial final o activa ( $G$ A $P_{f}$ ).

3.3. Grado de agresividad potencial real o efectiva ( $G A R$ ).

B. Tablas extraidas de dicha norma GTL 11357, con la que determinar cada uno de los conceptos indicados anteriormente, los cuales serán necesarios e imprescindibles para la resolución de los casos prácticos.

Tabla 1. "Tabla para la determinación de los G A $P_{i}$ y G A $P_{f}$ ".

Tabla 2." "Tabla para la determinación, o no, del G A R".

C. Tipo(s) de cemento(s), calidad del hormigón y/o medidas necesarias para cada caso concreto.

\section{A.1. Campos de utilización de la presente norma:}

a) Para confeccionar hormigón a pie de obra, o no.

b) Para confeccionar elementos prefabricados de hormigón, y

c) En general, para construir depósitos y demás productos de hormigón en los que este material se ponga o vaya a ponerse en contacto con terrenos HUMEDOS y/o aguas que contengan materiales o sustancias agresivas, como pueden ser:

- aguas muy puras de montaña y/o lluvia,

- aguas pantanosas,

- aguas agresivas subterráneas, superficiales o residuales,

- aguas residuales industriales,

- aguas de mar,

- terrenos correspondientes a las mismas.

\section{A.2. Examen}

\section{A.2.1. Toma de muestras del terreno y/o agua cuya agresividad para el hormigón se trata de determinar}

El examen y calificación de las muestras del terreno y/o agua para conocer sus propiedades agresivas para el hormigón, exige que en la extracción de las muestras y su conducción al laboratorio, se observen determinadas precauciones.

Una toma de muestra inapropiada:

- del terreno, puede introducir pérdidas en el contenido de $\mathrm{SFe}$ o $\mathrm{S}$, caso de que contenga ciertas incrustaciones de piritas $\mathrm{y} / \mathrm{o}$ azufre.

- del agua, puede alterar por ejemplo el contenido de carbónico y/o sulfhidrico en su caso.

Por lo tanto, y en general, se deben de observar las siguientes precauciones: 
$1^{\text {a }) ~ L o s ~ f r a s c o s, ~ p r o d u c t o s ~ q u i m i c o s ~ y ~ e n v a s e s ~ p a r a ~ e l ~ t r a n s p o r t e, ~ d e b e n ~ s o l i c i t a r s e ~ d e l ~ l a b o r a t o r i o ~}$ $o$ instituto de ensayos.

2. ${ }^{\mathrm{a}}$ ) La toma de muestra deberá de ser doble como mínimo.

3. $\left.{ }^{\mathrm{a}}\right)$ Para cada muestra del terreno son necesarios por lo menos $2.000 \mathrm{~cm}^{3}$.

4. $\left.{ }^{a}\right)$ El empaquetado y transporte de los envases con sus muestras respectivas se hará de modo y manera que no afecte en nada al contenido de la muestra envasada y evite su pérdida por o durante el transporte, remitiéndolas a continuación al lugar de su análisis y ensayo.

5. $\left.{ }^{a}\right)$ A veces puede ser necesario e imprescindible hacer el análisis en el mismo lugar de las tomas de muestras.

6. $\left.{ }^{a}\right)$ Para las tomas de muestras del terreno se debe de seguir lo especificado al efecto en la Norma DIN 4421, o NLT-101/72.

7. ${ }^{\text {a) }}$ Para las tomas de muestras de aguas se debe seguir lo especificado al efecto en alguna(s) de las siguientes normas existentes:

- Norma DIN 4000.

- Norma TGL 11456.

- Norma GOST 1030-41.

- Norma ME 35/63-C.

- Norma CSN 732020.

8. ${ }^{a}$ ) Tanto en un caso como en el otro, cuidese de que los envases sean de vidrio no soluble o resistente al agua del tipo correspondiente y al menos a la clase hidrolitica 2 según la norma DIN 12111.

\section{A.2.2. Análisis o determinaciones específicas de cada tipo de terrenos $y / o$ agua agresiva posible}

\section{ANALISIS DE TERRENOS:}

$\left.1^{\circ}\right)$ Para las determinaciones especificas se necesitan, como mínimo, $2.000 \mathrm{cc}$. del mismo.

2..$^{\circ}$ Sobre todo se deberán tomar las muestras del mismo para verificar su análisis correspondiente, cuando la capa freática no aparece pero existe la posibilidad de que ascienda hasta llegar a las cimentaciones de las obras.

3. ${ }^{\circ}$ Para poder juzgar un terreno es necesario e imprescindible investigar y determinar:

- el contenido de Materia Orgánica existente en el mismo (norma NLT-117/72).

- el contenido, en dicho terreno, de sales solubles en el agua o CLH (normas NLT-119/72 y NLT-120/72),

- el valor del $\mathrm{pH}$ del terreno, y si éste es menor de 6.5 debe investigarse el grado de acidez,

- el contenido de iones $\mathrm{SO}_{4}^{-}, \mathrm{Mg}^{2+}$ solubles en agua. 


\section{ANALISIS DE AGUAS:}

a) En general:

Deberán comprender, además de los datos de fecha, día, hora, temperatura y lugar de la toma de muestras, los siguientes datos:

* Aspecto.

* Color.

* Olor.

* Depósito, o no, de sales.

* Desprendimiento, o no, de burbujas.

* Resíduo de evaporación.

* Reacción ácida o alcalina.

* Dureza temporal y permanente.

* Consumo de $\mathrm{MnO}_{4} \mathrm{~K}$.

* Contenido de:

$-\mathrm{CaO}$.

- $\mathrm{CO}_{2}$ (total y agresivo para la cal).

$-\mathrm{Mg}^{2+}$.

$-\mathrm{NH}_{4}^{+}, \mathrm{Ca}^{2+}, \mathrm{Na}^{+}, \mathrm{K}^{+}$.

$-\mathrm{SO}_{4}^{=}, \mathrm{NO}_{3}^{-}, \mathrm{Cl}, \mathrm{S}^{=}$.

- y otras sustancias agresivas.
* Acidez total.

* Acidos minerales libres $\left(\mathrm{SO}_{4} \mathrm{H}, \mathrm{CLH}, \mathrm{NO}_{3} \mathrm{~K}\right.$, etc.).

* Acidos orgánicos (láctico, acético, etc.).

* Humus (ácidos húmicos y/o fúlvicos).

* Azúcar(es).

* Sulfatos solubles tales como $\mathrm{SO}_{4} \mathrm{Na}_{2}, \mathrm{SO}_{4} \mathrm{~K}_{2}$, $\mathrm{SO}_{4}\left(\mathrm{NH}_{4}\right)_{2}$, etc.

* Gases que contienen azufre, tales como $\mathrm{SH}_{2}$, $\mathrm{SO}_{2}$.

* Grasas y aceites vegetales y animales, sus ácidos grasos, así como los ácidos grasos sintéticos.

* Grasas y aceites minerales; contenido de resinas y ácidos.

* Materiales en suspensión.

* Oxígeno disuelto.

* Indice de Langelier.

b) En particular:

b.1. Para aguas de montaña. El análisis comprenderá preferentemente:

$* \mathrm{pH}$.

* $\mathrm{CO}_{2}$ agresivo a la cal.

* $\mathrm{CO}_{2}$ libre.

* $\mathrm{CO}_{2}$ combinado.

* Dureza temporal y permanente.

* Residuo evaporación.

b.2. Para aguas procedentes de pantano. Serán necesarias realizar, además, las siguientes determinaciones:

* Acidos orgánicos libres.

* Azufre.

* Sulfatos, $\mathrm{SO}_{4}^{-*}$.

* Amonio, $\mathrm{HN}_{4}^{+}$.

* Materiales en suspensión.

* Consumo de $\mathrm{MnO}_{4} \mathrm{~K}$.

* Cantidad de oxigeno disuelto. 
b.3. Para aguas residuales y residuales industriales. Serán necesarias realizar, además, las siguientes determinaciones específicas:

* Coloración más o menos oscura característica.

* Materiales en suspensión.

* Depósito de sales.

* Olor desagradable.

* Desprendimiento de burbujas.

* Reacción ácida o alcalina.

* Acidos minerales libres $\left(\mathrm{SO}_{4} \mathrm{H}_{2}^{\prime}, \mathrm{ClH}, \mathrm{NO}_{3} \mathrm{H}, \mathrm{SH}_{2}\right.$ principalmente).

* Acido carbónico.

* Acidos orgánicos (láctico, acético, succinico, etc.).

* Acido total.

* Sulfatos solubles en agua y en $\mathrm{ClH}$.

* Gases: $\mathrm{SH}_{2}$ y/o $\mathrm{SO}_{2}$ y sus disoluciones acuosas.

* Sales magnésicas y/o amónicas y/o sódicas y/o potásicas.

* Grasas, aceites, ácidos grasos y sus sales, en su caso.

b.4. Para aguas de mar. Primeramente y respecto a la toma de muestra, se debe de tener en cuenta que la misma se deberá realizar:

- a distintos niveles,

- en diferentes estaciones del año ya que la ta condiciona la atacabilidad del agua,

- anotando la fecha, lugar, profundidad, toma de agua de mar, ta ambiente, día lluvioso o soleado, etc.

y respecto a las determinaciones que en las mismas se deben de realizar se dividen en:

a) Principales o fundamentales:

* Sales magnésicas.

* Sulfatos.

* Cloruros.

Su importancia estriba en ser componentes muy notables en el agua de mar, y más aún los últimos.

b) Secundarios:

* $\mathrm{CO}_{2}$ agresivo para la cal.

$* \mathrm{pH}$.

A.3. Determinación de los distintos grados posibles de agresivos del terreno y/o agua problema en función de los diferentes datos de que se dispongan de los mismos

\section{A.3.1. Grado de agresividad Potencial Inicial o "Teórica" (G A $\left.P_{i}\right)$}

Para su determinación se compararán por separado, o no, según casos, cada uno de los resultados de los análisis obtenidos en A.2, con los valores numéricos de la Tabla 1, y se determinarán para cada uno y/o conjunto de ellos, según los casos, su $G A P_{i}$ respectivo, prevaleciendo sobre todos 
los demás aquel que dé mayor valor absoluto de la citada agresividad. Dicho valor, que será, como decimos, el superior a todos los demás obtenidos, será considerado, por razones de seguridad, como A.3.2. No obstante, si el terreno y/o agua problema tuviera dos o más sustancias con el mismo grado de agresividad, normalmente el $\mathrm{G} \mathrm{A}_{\mathrm{i}}$ se deberá incrementar en una unidad, empezando a ser ya A.3.2.

\section{A.3.2. Grado de Agresividad Potencial Final o "Activa" (G A $P_{f}$ )}

Como se ha dicho anteriormente, es el superior a todos los $\mathrm{G}$ A $\mathrm{P}_{\mathrm{i}}$ obtenidos de cada determinación o determinaciones analiticas concretas de A.2. Naturalmente, ya deberá estar incrementado en una unidad en el caso de que se cumpla el condicionamiento final del apartado anterior. A este $G$ A $P_{f}$ asi determinado y obtenido, le corresponde un hormigón de las siguientes condiciones:

1. a) El hormigón es de calidad B.120, donde:

$\mathrm{B}=$ el cemento apropiado escogido (se verá más adelante).

120 = número mínimo de $\mathrm{kp} / \mathrm{cm}^{2}$ del valor de la resistencia a compresión a los 28 días de edad, de una probeta cúbica de dicho hormigón B 120 , con $20 \mathrm{~cm}$ de arista.

2. ${ }^{\text {a) }}$ El hormigón está enterrado en terreno permeable de arena o grava.

$\left.3 .^{a}\right)$ Se supone que se trata de agua en reposo, en contacto con hormigón aún no endurecido hasta la calidad antes indicada.

\section{A.3.3. Grado de Agresividad Real o "Efectiva" (G A R)}

Se obtiene a partir del valor del $\mathrm{G} \mathrm{A} \mathrm{P}_{\mathrm{f}}$ incrementándolo o reduciéndolo en función de las condiciones "locales", o no, dadas en la Tabla 2 y obtenidas por la experiencia.

Este valor del G A R deberá determinarse por el proyectista en función de las condiciones y/o características "locales" de "su obra" proyectada, las cuales él mejor que nadie deberá de conocer.

\section{Notas adjuntas al apartado A.3.}

1. Si las circunstancias "locales" no coinciden con las cifras expuestas en las Tablas 1 y 2 , el proyectista valorará el G A $\mathrm{P}_{\mathrm{i}}$ y el G A R por otros medios o normas.

2. Las indicaciones dadas en la Tabla 2 proporcionan sólamente orientación de como se podria conseguir el G A R.

3. Se elevará una consulta previa a los organismos facultados para ello, o a los técnicos autorizados oficialmente, sobre las precauciones pertinentes frente a la A.R.* cuando:

3.1. El G A R del agua problema sea simplemente o "muy fuertemente agresiva para el hormigón", o con un $\mathrm{pH}<3$, o con un contenido de sustancias que sobrepase el doble del valor que aparece en la Tabla 1.

3.2. El G A R para el hormigón sea "muy fuertemente agresivo".

3.3. El G A P del agua problema, según esta norma TGL 11357 no aparezca determinado con seguridad y exactitud.

\footnotetext{
* A.R.-Agresividad Real.
} 
T ABLA 1

Agresividad del agua debida a las sustancias disueltas. TGL 11357

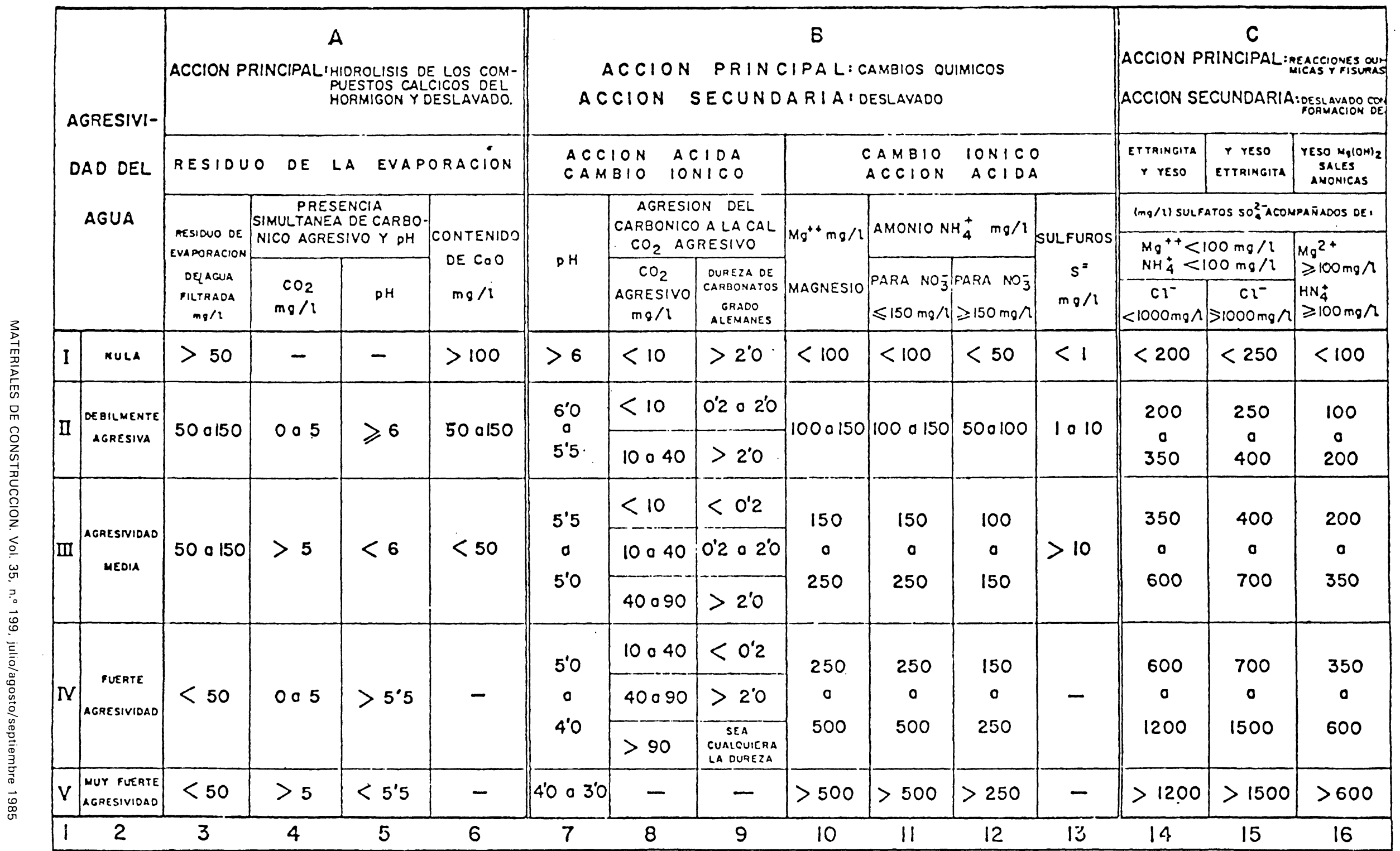




\section{B. TABLAS EXTRAIDAS DE LA NORMA TGL 11357}

B.1. Tabla 1. "Tabla para determinación de los G A P"

B.2. Tabla 2. "Tabla con los datos locales*, o no, necesarios en su caso para la determinación del G A R".

TABLA 2

\begin{tabular}{|c|c|}
\hline$+\mathbf{I}$ & $-\mathbf{I}$ \\
\hline $\begin{aligned} &+ \text { Si el terreno y/o agua problema posee dos o más } \\
& \text { sustancias disueltas y reaccionantes entre si con el } \\
& \text { mismo G A P, } \mathrm{NO}_{3}^{-} \text {y } \mathrm{NH}_{4}^{+}, \mathrm{SO}_{4}^{=} \mathrm{y} \mathrm{Mg}^{2+}, \mathrm{Cl}^{-1} \text { y } \\
& \mathrm{Ca}^{2+} \text {, etc. } \\
&+ \text { Agua problema corriente o fluyente (en movimiento). } \\
&+ \text { Nivel acuoso variable. } \\
&+ \text { Presión hidráulica unilabral. } \\
&+ \text { Temperatura del agua problema mayor de } 45^{\circ} \mathrm{C} . \\
&+ \text { Elemento de hormigón de sección reducida, o sea, } \\
& \text { estructuras laminares de hormigón, micropilotes, } \\
& \text { etcétera. }\end{aligned}$ & $\begin{array}{l}\text { - El agua agresiva para el hormigón entra en con- } \\
\text { tacto con él pasados } 28 \text { dias de su fabricación. } \\
\text { - El agua agresiva sólo se pone en contacto con la } \\
\text { obra algunas veces al año (por crecidas o riadas) } \\
\text { y el terreno es de tipo suelto, no cohesivo. } \\
\text { - El hormigón se encuentra rodeado por terrenos } \\
\text { cohesivos o compactos, tales como arcilla, ba- } \\
\text { rro, fango arcillosos, margas arcillosas, margas } \\
\text { glaciares, etc.; pues bien, la reducción en este } \\
\text { caso puede tener distinto valor según sea el IN- } \\
\text { DICE DE PLASTICIDAD Wfa, del terreno en } \\
\text { cuestión, asi tenemos que: } \\
\quad \text { Si } 0.05<\text { Wfa } * *<0,10 \rightarrow-\text { I } \\
\quad \text { Si Wfa } \geq 0,10 \rightarrow-\text { II } \\
\text { - Distintas operaciones finales que mejoren la imper- } \\
\text { meabilidad del hormigón tales, como pintura, bitu- } \\
\text { minosos, ocratado, etc. }\end{array}$ \\
\hline
\end{tabular}

* Condiciones "locales" dadas u obtenidas por la experiencia.

** Indice de plasticidad. Wfa = limite liquido (Wf) - limite plástico (Wa). (Veánse normas NLT-105/72 y NLT 106/72).

Las indicaciones dadas en la Tabla 2, son sólo datos indicativos para la deducción del G A R para el hormigón. De aqui que, en cualquier caso, sea conveniente obtener el dictamen confirmativo del mismo de alguno(s) de los centros especializados.

\section{CALIDAD DEL HORMIGON Y MEDIDAS DE PROTECCION}

En el apartado A.3.2. ya se habló de las condiciones y características que debe reunir un hormigón para satisfacer el $G$ A $P_{f}$. No obstante y puesto que en la realidad las condiciones "locales" de la obra proyectada pueden influir notoriamente sobre aquella, habrá de ser finalmente sobre el G A R correspondiente a cada caso concreto, sobre el que habrán de fijarse las condiciones y caracteristicas del hormigón necesario para resistirlo. Así tendremos que:

\section{C.1. Para G A R $=\mathrm{I}=$ AGUA NO AGRESIVA:}

No son necesarias medidas especificas.

\section{C.2. Para G A R = II = AGUA DEBILMENTE AGRESIVA:}

Por lo menos el hormigón deberá ser de la clase B 150, siendo necesaria la utilización de un conglomerante adecuado para cada caso concreto. 
Asi tendremos que:

a) Si la débil agresividad del terreno y/o agua agresiva, es debida fundamentalmente al $\mathrm{CO}_{2}$ agresivo a la cal, sin la adicional posible de iones sulfato, se recomiendan los siguientes cementos:

* Cementos Puzolánicos, de matriz clinker portland. No obstante la cantidad añadida de puzolana no será tenida en cuenta como conglomerante para el cálculo de la relación agua/cemento, a/c, tanto en este caso de agresividad como en cualquier otro.

* Cementos de Escorias Siderúrgicas*, preferentemente ácidas, de matriz clínker portland y cuyo contenido de escoria debe ser el máximo tolerable, pudiendo oscilar entre el $30 \%$ y el $80 \%$ en peso. No obstante estos últimos están recomendados preferentemente para hormigón en masa, pues en el armado existe posible peligro en su caso, de corrosión de armaduras, por el $\mathrm{S}^{=}$de la escoria, cuya cantidad está limitada en las normas por tal motivo, además de por la posible formación de $\mathrm{SO}_{4}=$ agresivo tras su oxidación correspondiente (escorias "expansivas").

* Además se toleran los cementos:

- Portland de bajo contenido de $\mathrm{C}_{3} \mathrm{~S}$,

- Portland con adiciones activas de cualquier tipo, hasta un total de $20 \%$ en peso, y

- Portland con escorias, preferentemente ácidas, hasta un total del $30 \%$ en peso,

siempre que el agua problema entre en contacto con el hormigón después de pasados, al menos, 28 dias de su puesta en obra, es decir, el agua problema no debe entrar en contacto con el hormigón fresco.

* Cementos Aluminosos, con las precauciones que se describen al respecto en el apartado siguiente b).

- El árido deberá ser silicico no reactivo (ASTM C 227-71 de 1976 y ASTM C 289-71 Part. 14, 1978).

b) Si la débil agresividad del terreno y/o agua agresiva, es debida fundamentalmente a los iones sulfato, con o sin la adición posible del $\mathrm{CO}_{2}$ agresivo para la cal, se recomiendan LOS CEMENTOS DE ELEVADA RESISTENCIA AL ATAQUE DE LOS IONES SULFATO, como pueden ser:

* Cementos Portland de contenido de $C_{3} A$ menor del $5 \%, y$ contenido $C_{4} A F$ también limitado a veces, aunque esta limitación difiere de unos paises a otros: entre ellos están los cementos tipo V, según la norma ASTM C 150-84 y similares, tales como "Ferrari”, Kühl, Hidrotécnico, PY, RPC, SRC, etc...

* Cementos Puzolánicos de matriz clínker portland de escaso, menos del $5 \%$, a nulo contenido de $C_{3} A$ preferentemente, y sobre todo PUZOLANA ADECUADA. Y si no se dispusiera de tal tipo de clinker, pudiera valer algún otro de mediano contenido de $\mathrm{C}_{3} \mathrm{~A}$, del $5 \%$ al $7 \%$ u $8 \%$, siempre y cuando que LA PUZOLANA SEA LA ADECUADA A ESTE CASO, lo cual no implica que obligatoriamente tenga que ser la misma que la primera.

* O siderometalúrgicas. 
* Cementos de ESCORIAS SIDERURGICAS* ADECUADAS, ya sean ácidas o básicas, de matriz clinker portland de escaso, menos del $5 \%$ a nulo contenido de $\mathrm{C}_{3} \mathrm{~A}$ preferentemente, y cuyo contenido de aquellas puede oscilar entre el $50 \%$ y el $80 \%$ en peso, aunque en ocasiones quizás pudiera descender al $30 \%$ en peso, según el grado de adecuación de dicha escoria siderúrgica. Y si no se dispusiera de tal tipo de clínker, pudiera valer algún otro de mediano contenido de $\mathrm{C}_{3} \mathrm{~A}$, del $5 \%$ al $7 \%$ u $8 \%$, siempre y cuando la ESCORIA SEA LA ADECUADA A ESTE CASO.

No obstante, se ha de saber que, en general, estos tipos de cementos de escorias siderúrgicas están especialmente recomendados para hormigón en masa, pues en el armado existe posible peligro, en su caso, de corrosión de las armaduras por el $\mathrm{S}^{=}$de la escoria cuya cantidad está limitada en las normas, por tal motivo, además de por la posible formación de $\mathrm{SO}_{4}^{=}$agresivo tras su oxidación correspondiente (escorias "expansivas").

\section{* CEMENTOS SOBRESULFATADOS}

- para aguas que contengan hasta $1.200 \mathrm{mg} / l$ de $\mathrm{SO}_{4}^{=}$; no obstante, si simultáneamente existen en el agua más de $600 \mathrm{mg} / l$ de $\mathrm{Mg}^{2+} \mathrm{y} / \mathrm{o} \mathrm{NH}_{4}^{+}$, este cemento es menos apropiado.

- su hormigón fresco o recién endurecido, no debe ponerse en contacto con agua que contenga $\mathrm{CO}_{2}$ agresivo para la cal, o cuyo $\mathrm{pH}<6$.

* CEMENTOS ALUMINOSOS: En algunos casos especiales, pueden emplearse los cementos aluminosos frente a las aguas sulfatadas, cuando no existen sales de $\mathrm{NH}_{4}^{+}$, álcalis, o temperaturas del agua problema elevadas.

* $\mathrm{Y}$ en su defecto, quizás los de MODERADA O MEDIANA RESISTENCIA AL ATAQUE DE LOS IONES SULFATO, según la norma ASTM C 150-84 a cemento tipo II (USA) y similares.

\section{RECOMENDACION}

Sea cual fuere el cemento puro o de mezcla elegido, en especial a este último, se le deberá confirmar previamente, antes de su utilización en obra, en un laboratorio especializado y mediante el método acelerado de ensayo adecuado al mismo, su grado adecuado de resistencia al ataque de los iones sulfato.

- El árido podrá ser silícico no reactivo o calizo.

$\mathrm{Y}$ finalmente, en el supuesto de que no se logre disponer del cemento recomendado, la calidad del hormigón deberá ser por lo menos B 160 , con una relación $\mathrm{a} / \mathrm{c}<0,65$, o dosificaciones de cemento superiores a $300 \mathrm{~kg} / \mathrm{m}^{3}$.

\section{C.3. Para G A R = III = AGUA DE AGRESIVIDAD MEDIA}

- La granulometria del árido empleado debe encontrarse entre las líneas de cribado $\mathrm{E}$ y $\mathrm{F}$.

* O Siderometalúrgicas. 
- La calidad del hormigón preparado, por lo menos será B 160 con una relación agua/cemento menor que 0,65 , o contener como mínimo $300 \mathrm{~kg}$ de cemento por $\mathrm{m}^{3}$ de hormigón.

- Es necesario la utilización de un conglomerante adecuado para cada caso concreto. Así tenemos que:

a) Si la agresividad media del terreno y/o agua problema, es debida fundamentalmente al $\mathrm{CO}_{2}$ agresivo para la cal, sin la adicional posible de iones sulfato, se recomiendan los siguientes cementos:

* Cementos Puzolánicos, de matriz clinker portland,

* Cementos de Escorias Siderúrgicas*, preferentemente ácidas, de matriz clínker portland y cuyo contenido de escoria debe ser el máximo tolerable pudiendo oscilar entre el $30 \%$ y el $80 \%$ en peso.

En cualquier caso el terreno y/o agua problema no deberán entrar en contacto con el hormigón fresco o recientemente endurecido, debiendo dejar pasar, como mínimo, 28 días hasta que se produzca aquel. En caso contrario y necesario, podría sobredimensionarse la estructura con un recubrimiento calizo o con un hormigón de cemento portland o portland con adiciones activas, a fondo perdido, sobre la estructura fundamental de aquel que tratamos de preservar a edades iniciales.

- El árido deberá ser silícico no reactivo.

b) Si la agresividad del terreno y/o agua agresiva es debida fundamentalmente a los iones sulfato, con o sin, la adicional posible del $\mathrm{CO}_{2}$ agresivo para la cal, se recomiendan LOS CEMENTOS DE ELEVADA RESISTENCIA AL ATAQUE DE LOS IONES SULFATO, como pueden ser:

* Cementos Portland de contenido de $C_{3} A$ menor del $5 \%$, y contenido de $\mathrm{C}_{4} \mathrm{AF}$ también limitado, a veces, aunque esta limitación difiere de unos paises a otros: entre ellos están los cementos tipo V, según la norma ASTM C150-84a y similares, tales como "Ferrari", Kühl, Hidrotécnico, PY, RPC, SRC, etc.

* Cementos Puzolánicos de matriz clínker portland de escaso, menos del $5 \%$, a nulo contenido de $C_{3} A$, preferentemente, y sobre todo PUZOLANA ADECUADA. Y si no se dispusiera de tal tipo de clinker, pudiera valer algún otro de mediano contenido de $\mathrm{C}_{3} \mathrm{~A}$, del $5 \%$ al $7 \%$ u $8 \%$, siempre y cuando la PUZOLANA SEA LA ADECUADA A ESTE CASO, lo cual no implica que obligatoriamente tenga que ser la misma que la primera.

* Cementos de ESCORIAS SIDERURGICAS* ADECUADAS, ya sean ácidas o básicas, de matriz clinker portland de escaso, menos del $5 \%$, a nulo contenido de $\mathrm{C}_{3} \mathrm{~A}$, preferentemente, y cuyo contenido de aquellas, puede oscilar entre el $50 \%$ y el $80 \%$ en peso, aunque en ocasiones quizás pudiera descender al $30 \%$ en peso, según el grado de adecuación de dicha escoria siderúrgica. $\mathrm{Y}$ si no se dispusiera de tal tipo de clínker, pudiera valer algún otro de mediano contenido de $\mathrm{C}_{3} \mathrm{~A}$, del $5 \%$ al $7 \%$ u $8 \%$, siempre y cuando la ESCORIA SEA LA ADECUADA A ESTE CASO.

* Cementos Aluminosos con las precauciones descritas en el punto C.2.b).

* O Siderometalúrgicas. 
* Cementos sobresulfatados, con las precauciones descritas en el punto C.2.b).

Y por otra parte, en el caso de empleo de cemento sobresulfatado o aluminoso, no se deberá olvidar que el agua empleada para el amasado del hormigón, debe ser de características análogas al agua potable.

\section{RECOMENDACION}

Sea cual fuese el cemento puro o de mezcla elegido, en especial a este último, se le tendrá que confirmar previamente, antes de su utilización en obra, en un laboratorio especializado y mediante el método acelerado de ensayo adecuado al mismo, su elevada resistencia al ataque de los iones sulfato.

- El árido podria ser silícico no reactivo o calizo.

\section{C.4. Para G A $\mathrm{R}=\mathrm{IV}=$ AGUA DE AGRESIVIDAD FUERTE:}

- La granulometría del árido debe estar en una zona especialmente favorable, entre las líneas de cernido D y E.

Los áridos se suministrarán en tres tamaños (de 0 a $2,5 \mathrm{~mm}$; de 2,5 a $5 \mathrm{~mm}$ y más de $5 \mathrm{~mm}$ ), habiéndose de mezclar mecánicamente, teniendo que ser del tipo indicado en C.2 y C.3. para cada caso concreto.

- La relación a/c $<0,45$ y en el hormigonado bajo agua, el valor de esta relación será, como máximo de 0,4 .

- Estas condiciones conducen mediante la utilización de un cemento elegido medio Z-225, con una relación $\mathrm{a} / \mathrm{c}=0,45$ y una cantidad de aire menor de $1,5 \%$, a una calidad de hormigón aproximadamente B 300 .

- La cantidad de cemento elegido viene condicionada por la granulometria de los áridos y la consistencia impuesta por su compacidad, de modo y manera que para canto rodado de tamaño inferior a 25 milimetros será de 350 a $450 \mathrm{Kg}$ de cemento por metro cúbico de hormigón.

- El cemento $\mathrm{Z}$ será alguno(s) de los recomendados en el apartado anterior C.3., según cada caso, a) o b), posible.

- Si no se dispone del tipo de cemento adecuado al caso, se adoptarán las medidas de protección descritas en el apartado siguiente C.5.

- El hormigón fresco puede protegerse antes de ser atacado por el agua agresiva, mediante pinturas a base de betún o alquitrán en forma de disoluciones o emulsiones (norma TGL 3652) o mediante fluatación.

- Se comprobará la impermeabilidad del hormigón a los 90 días en la forma adecuada. Para ello una vez alcanzada una presión hidráulica de $7 \mathrm{kp} / \mathrm{cm}^{2}$, la penetración del agua no debe ser superior a $80 \mathrm{~mm}$, pudiendo estimarse la impermeabilidad adecuada cuando en el hormigón fresco el volumen de poros es inferior al $1,5 \%$. 
esto se cumple cuando:

$$
\frac{Z}{\rho_{0_{\mathrm{z}}}}+\frac{K}{\rho_{\mathrm{k}}}+W=985 \mathrm{dm}^{3}
$$

siendo:

$\rho_{0_{\mathrm{z}}}=$ Densidad real del cemento empleado.

$\rho_{\mathrm{k}}=$ Peso de 1 litro del árido total.

$Z=$ Dosificación de cemento.

$W=$ Cantidad de agua de amasado.

$K=$ Cantidad de árido.

Los tres últimos conceptos se darán en $\mathrm{kg} / \mathrm{m}^{3}$ de hormigón fresco compactado, sin adición de aireantes.

El contenido de poros puede determinarse mediante el cálculo del volumen sólido o mediante ensayo. Estas medidas previas no implican la eliminación de la comprobación de la impermeabilidad.

\section{C.5. Para G A $\mathbf{R}=\mathbf{V}=$ AGUA DE AGRESIVIDAD MUY FUERTE:}

Se mantienen las prescripciones del apartado anterior C.4. Además se debe ensayar la permeabilidad para el agua con $7 \mathrm{kp} / \mathrm{cm}^{2}$ de presión hidráulica y la profundidad de penetración sólo debe alcanzar a $40 \mathrm{~mm}$. Para asegurar la impermeabilidad se adicionarán las siguientes cantidades mínimas de elementos finos $(0$ a $2 \mathrm{~mm})$ del árido y de piedra molida $(0$ a $0,2 \mathrm{~mm})$ por cada metro cúbico de hormigón.

\begin{tabular}{|c|c|}
\hline Para áridos de tamaño de: & $\mathbf{K g}$ \\
\hline 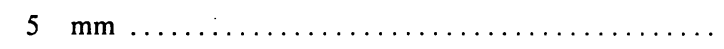 & 500 \\
\hline $12,5 \mathrm{~mm}$ & 425 \\
\hline $25 \mathrm{~mm}$ & 350 \\
\hline $35,5 \mathrm{~mm} \ldots$ & 300 \\
\hline $56 \mathrm{~mm}$. & 225 \\
\hline
\end{tabular}

- Los áridos habrán de ser del tipo indicado en C. 2 y C. 3 para cada caso concreto.

- El volumen de poros del hormigón fresco compactado no será superior al $1 \%$ para que se cumplan los requisitos referentes a impermeabilidad. Las armaduras tendrán recubrimientos superiores a $50 \mathrm{~mm}$.

Podrán emplearse adiciones de puzolanas ADECUADAS para cada caso concreto, debiéndose de confirmar previamente en laboratorios especializados su buen comportamiento. Igualmente podrán emplearse modificadores del fraguado tales como plastificantes, aireantes, impermeabilizantes en masa.

Las medidas de protección citadas son aplicables a los hormigones expuestos a la acción del agua de mar. 
Si las medidas expuestas no pareciesen suficientes habrá de cuidarse que el agua no se ponga en contacto con el hormigón. Esto puede realizarse, por ejemplo, adoptando medidas constructivas especiales, drenaje del agua o descenso de su nivel, impermeabilizando según la técnica correspondiente mediante junturas impermeabilizantes, materias plásticas, revestimiento de clínker, impermeabilizantes, con arcilla o con bandas protectoras (normas TGL 3651 y 3653).

\section{ESPECIFICACIONES ADICIONALES}

Los tubos de hormigón fraguado y pretensado han demostrado ser resistentes a las aguas fuertemente agresivas, si su fabricación responde a las normas.

El recubrimiento de $30 \mathrm{~mm}$ de hormigón de las armaduras puede ser reducido en estos casos. En las aguas muy intensamente agresivas, deben tomarse las oportunas medidas de protección para armaduras y hormigón.

\section{B I B L I O G R A F I A}

(1) I. BICZOK: La corrosión del hormigón y su protección. Ed. Urmo, C/ Espartero, 10. Bilbao. España 1972.

(2) PLIEGO DE CONDICIONES PARA LA RECEPCION DE CONGLOMERANTES HIDRAULICOS. PCCH-64, 3. ${ }^{a}$ ed. Madrid, 1972.

(3) PLIEGO DE PRESCRIPCIONES TECNICAS GENERALES PARA LA RECEPCION DE CEMENTOS. RC-75. MOP Secretaria General Técnica, Servicio de Publicaciones, Madrid.

\section{publicación del i.e.t.c.c.}

\section{bases para el diseño solar pasivo}

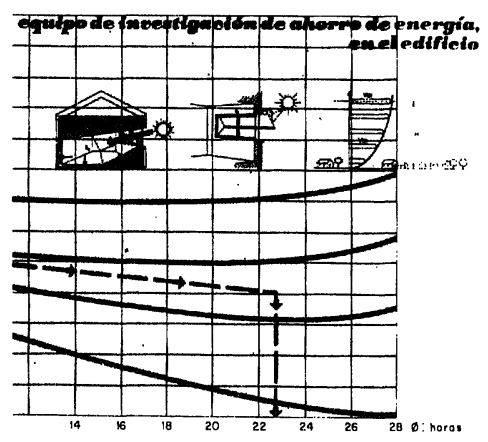

Instituro Eduardo Torroje de la Construcción y del Camanto
CONSEJO SUPERIOA OE INVESTIGACLONES CIENTIFICAS
Equipo de Ahorro de Energia en el edificio

Dirección y coordinación:

Arturo Garcia Arroyo

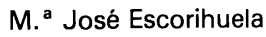

José Luis Esteban

José Miguel Frutos

Manuel Olaya

Bernardo Torroja
Las dificultades de suministro y el alto coste de los productos energéticos convencionales han despertado la atención de los usuarios, técnicos e industriales de la edificación hacia los procedimientos y sistemas en que se basa el aprovechamiento de otras fuentes alternativas de energia, principalmente la solar. Esto ha generado un rápido desarrollo industrial $y$ comercial que, en opinión de los autores de come libro que, en este libro, arrastran los siguientes defos: los sistemas convencionales que violenta las peculiaridades de la energia solar (baja densidad y variabilidad en el tiempo), y una escasa selectividad en la aplicación de los sistemas y procedimientos pasivos dando origen a un ecumenismo arquitectónico solar, al margen de las condiciones climáticas y funcionales específicas de cada caso y lugar.

En este libro, utilizando criterios y metodologia pedagógicos, se dan los fundamentos e instrumentos teórico-prácticos necesarios para el planteamiento de todo proyecto arquitectónico solar pasivo, de acuerdo con los principios éticos y económicos de conservación y ahorro de enérgia. Es decir: respeto de los presupuestos bioclimáticos, búsqueda de la máxima captación y acumulación de la radiación solar, y esmero en el aislamiento térmico de los cerramientos.

Un volumen encuadernado en cartulina ibiza plastificada, a cinco colores, de $16 \times 23 \mathrm{~cm}$, compuesto de 216 páginas, 217 figuras, 87 gráficos, 19 tablas y 10 cuadros.

Madrid, 1983. Precios: España 2.100 ptas.; 30 \$ USA. 